\title{
Tuning of localized surface plasmon resonance in copper-copper oxide core-shell quantum dots for the application in biosensors
}

\author{
Prabhash Prasannan Geetha, Ajith Ramachandran, Swapna S. Nair* \\ Department of Physics, Central University of Kerala, Kasaragod, Kerala - 671 314, INDIA
}

\begin{abstract}
Surface Plasmon Resonance (SPR) is an attracting property of certain transition metals when they are synthesized in nano-range giving rise to promising optical applications. However, most SPR and associated applications are limited to the noble metal nanoparticles, which limits their potential due to high production cost. We report surface plasmon resonance in copper-copper oxide core-shell quantum dots synthesized via chemical route studied by using UV-Visible spectrophotometry. Tuning of the plasmonic resonance with respect to the particle diameter is achieved by an inexpensive all chemical route. Photoluminescence measurements also support the data. This size reduction leads to remarkable changes in its optical response as compared to the bulk metal. The results point towards applications of these materials in tunable SPR based biosensors.
\end{abstract}

Keywords: Copper, Surface Plasmon Resonance, Bio-sensor

\section{INTRODUCTION}

Metal nanoparticles have fascinated scientists for over a century and are the focus of interest because of their huge potential in nanotechnology. Nowadays several synthesis techniques are being developed so as to modify these properties. The metal nanoparticles show good compatibility, catalytic behaviour, and SurfaceEnhanced Raman activity [1-4]. Noble metal nanoparticles in general and their gold and silver analogue, in particular, attract huge research interest owing to their fabulous properties (electrical and optical) and diversity of applications including fluorescence imaging, biomedical applications and bio-engineering. Mankind has been ensnared by noble metals (gold and silver) since prehistoric times and applications of their nanoparticles have attracted attention for the last millennia, although understanding many of the phenomena at the nanoscale is very recent. Nanoscale analogues are being explored due to their unusual functional attributes quite unlike the bulk [5-8]. As research in this area moves forward in nanomaterials, scientists are discovering novel application possibilities. Tunability of nanoparticle properties is possible by varying size, shape, composition or local environment present in them. By manipulating the chemical composition of materials at the nanoscale, their chemical, optical, electrical and other properties can be tailored precisely $[7,9,10]$, which makes them ideal from an application point of view.

Noble metal nanoparticles have large optical field enhancement resulting in strong scattering and absorption of light. The enhancement of the optical properties of noble metal nanoparticles arises due to resonant oscillations of their free electrons in the presence of light and this is known as localized surface plasmon resonance [LSPR] $[1,11]$. The localized surface plasmon resonance is the result of the confinement of surface plasmon in the nanoparticle of a size comparable to or smaller than the wavelength of light used to excite the plasmon. Local-

* Swapna S Nair: swapna.s.nair@gmail.com ized surface plasmon has two effects, i) the electric fields near the particle surface are greatly enhanced, which falls-off quickly with distance from the surface. ii) The optical extinction of nanoparticles is the maximum at the surface plasmon resonant frequency and this occurs at a visible wavelength for the noble metal nanoparticles, which enhances their applications in various fields $[1,2,5,6,9,12,13]$.

Plasmon resonant frequency is highly sensitive to the index of refraction of the environment. This allows nanoparticles possessing localized surface plasmon to be used for nanoscale sensing application [11, 14, 15]. Nanostructures exhibiting localized surface plasmon resonances are used to enhance the optical signals in modern analytical techniques based on spectroscopy.

The plasmon resonance can either radiate (Mie scattering) light, which is useful in optical and imaging fields or is converted into heat(absorption) which has opened up applications in several areas [16-18]. Nanoparticles with SPR has the ability to integrate metal nanoparticles into the biological systems to have live fluorescence imaging and thus contributes much into the area of biology and biomedicine.

Most of the experimental studies on surface plasmons are limited to noble metals. However, in this work, we propose that copper can also emerge as a promising SPR candidate [19-22].

\section{EXPERIMENTAL TECHNIQUES}

Synthesis: Copper quantum dots were prepared by chemical reduction method using Copper (II) Chloride $\left(\mathrm{CuCl}_{2}\right)$, Sodium Borohydride $\left(\mathrm{NaBH}_{4}\right)$. Citric acid $\left(\mathrm{C}_{6} \mathrm{H}_{8} \mathrm{O}_{7}\right)$ and $\mathrm{CTAB}$ (Cetrimoniumbromide$\mathrm{C}_{19} \mathrm{H}_{42} \mathrm{BrN}$ ) were used as surfactants. 0.01M Copper chloride was added to $0.01 \mathrm{M}$ solution of citric acid. $0.5 \mathrm{M}$ freshly prepared ice-cold $\mathrm{NaBH}_{4}$ was added by n drops ( $\mathrm{n}=10,20,30,40$, excess drops) into the solution, to yield copper quantum dots. The surface of the copper nanoparticles was subsequently oxidized to form a layer of copper oxide. The experiment is repeated using CTAB instead of citric acid crystals. 
The samples were analyzed using X-Ray Diffraction in Rigaku D Max Miniflex X-Ray Diffractometer with copper $K_{\alpha}$ line $(1.5406 \AA)$ as the wavelength. The lattice planes were identified and indexed. The lattice parameter was measured. Microstructural properties were probed via SEM (Hitachi SU 6600 scanning electron microscope, Horiba Emax EDS). The elemental identification was done using Energy Dispersive X-Ray analysis (EDX). The particle size was measured using Transition Electron Microscopy (JEOL JEM 2100F) and the lattice planes were identified using Selected Area Electron Diffraction (SAED). High-resolution transmission electron microscopy (HRTEM) was used to see the lattice of the quantum dots.

Optical characterisation of copper quantum dots was carried out by using UV-Visible spectrophotometer (PerkinElmer UV-Vis Spectrophotometer, DRS). Absorbance was measured as a function of wavelength from 200 to $900 \mathrm{~nm}$ in order to SPR. Photoluminescence spectroscopy (PerkinElmer LS 55 Fluorimeter) was used to further probe into the optical characteristics.

Theoretical studies: The extinction spectra for the copper nanoparticles were theoretically studied using Finite Element Method based software COMSOL Multiphysics [23]. The material parameters of the copper nanoparticles were taken from Rakic et.al. based on the Brendel-Bormann (BB) model [24]. The nanoparticle was considered to be immersed in water (refractive index $=1.33)$. It was assumed that the nanoparticle was illuminated by a plane wave source in order to excite all resonance modes of the particle within the visible and IR region of the spectrum. The computational domain was truncated using a perfectly matched layer to avoid any spurious reflection from domain boundary [25].

\section{RESULTS AND DISCUSSION}

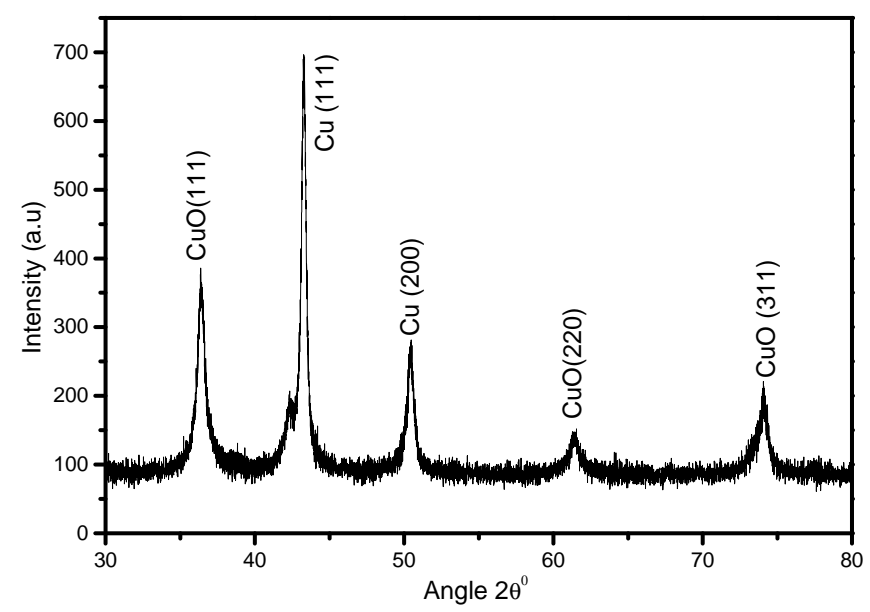

FIG. 1. XRD spectra of copper-copper oxide core-shell quantum dots.

Structural and morphology analysis: The XRD analysis was carried out to investigate the crystal phase and structure of copper-copper oxide core-shell nanocomposite. The X-ray diffraction patterns of core-shell nanocomposites were shown in Fig. 1 and the obtained peaks were compared with standard JCPDS ICDD data and the sample was found to be in cubic structure. The peaks exactly match with the JCPDS ICDD data (JCPDS no: 03-1005 for copper and 78-0428 for copper oxide) and the obtained planes corresponding to the peaks for copper are (111) and (200) and copper (II) oxide are (111), (220) and (311) respectively. Out of these, the most intense peak corresponding to the copper is (111). The average lattice constant is found to be $3.616 \AA$ for copper, and $4.259 \AA$ for copper oxide, which is found to be a slight variation from the standard value (for copper $\mathrm{a}=3.610 \AA$ and for copper oxide $\mathrm{a}=4.683 \AA$ ). The lattice parameter is found to be increased for copper and decreased for copper oxide, due to the lower grain size and larger surface to volume ratio and the negative surface stress (Pressure/surface energy induced effects Laplace's law in thermodynamics). The average lattice strain is found to be 0.005265 for copper and 0.00840 for copper oxide. Form the detailed analysis, we can confirm that the ultrafine copper nanoparticles were formed initially but subsequently oxidized its surface to form a highly crystalline copper oxide shell.
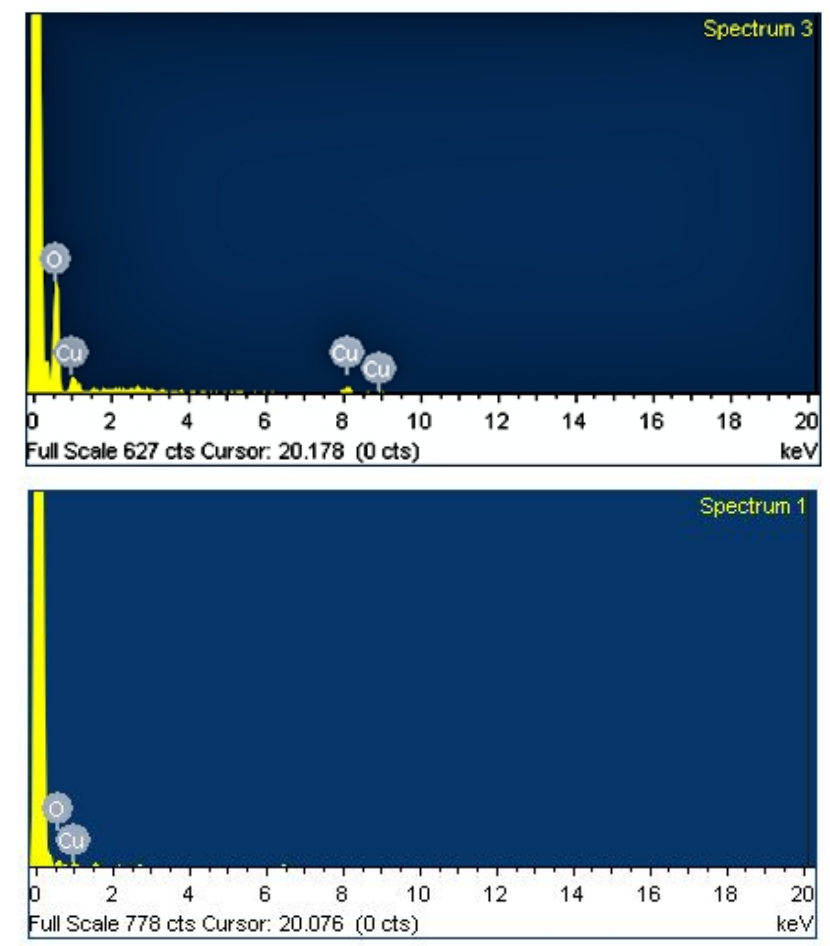

FIG. 2. EDX spectra of copper-copper oxide core-shell quantum dots. The peaks of quantum dots are very weak because the nanoparticles are in the confinement regime. (a) represents EDX of citric acid coated copper-copper oxide coreshell quantum dots, (b) represents the EDX of CTAB coated copper-copper oxide core-shell quantum dots.

From the EDX analysis (Fig. 2), it was observed that 

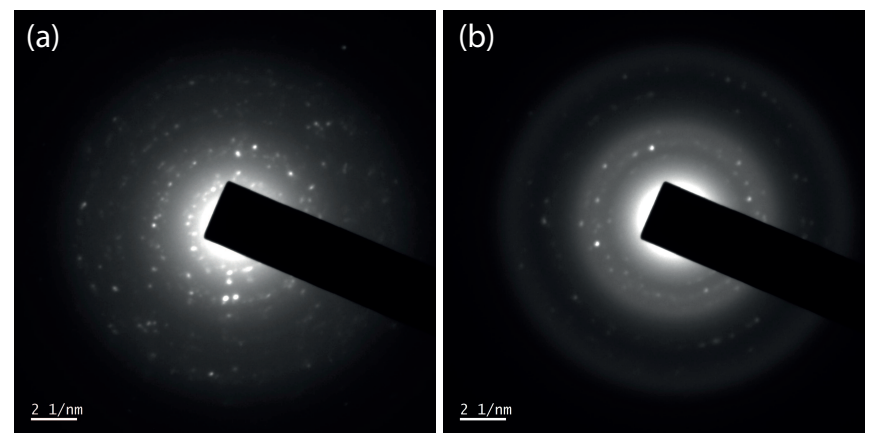

FIG. 4. Selected Area Electron Diffraction (SAED) images of copper-copper oxide core-shell quantum dots. (a) represents the citric acid coated copper-copper oxide core-shell quantum dots , (b) represents the CTAB coated copper-copper oxide core-shell quantum dots.

the solution contains spherical nanoparticles only. When the amount of the reducing agent was increased, the percentage of copper in the medium also increased, contributing to the higher particle density in the colloid. Employment of ice-cold reducing agent helps to synthesize the nanoparticles with very small particle diameter: because at low temperature, the kinetic energy of the $\mathrm{H}^{+}$ ions are very low, which helped to the formation of ultrafine nanoparticles [26]. The SEM images show good surface morphology of nanoparticles (Fig. 3). Selected area electron diffraction studies (Fig. 4) also confirmed the presence of copper-copper oxide quantum dots.
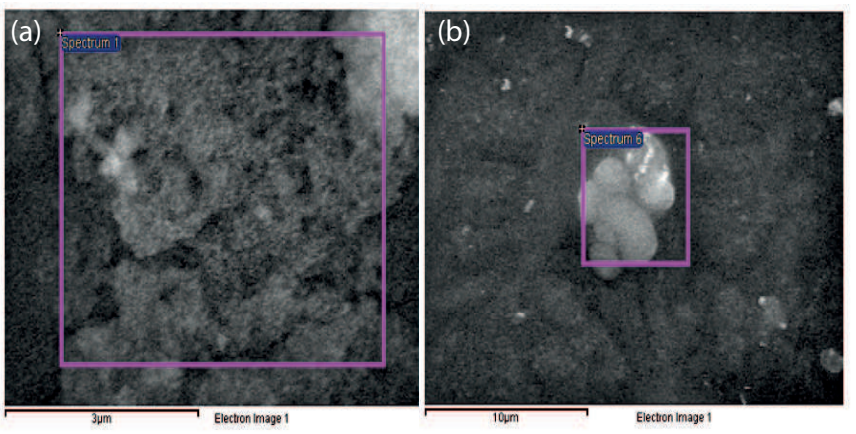

FIG. 3. The SEM images of copper-copper oxide core-shell quantum dots. It can be seen that the nanoparticles particles show very good surface morphology. (a) represents the citric acid coated copper-copper oxide core-shell quantum dots, (b) represents the CTAB coated copper-copper oxide core-shell quantum dots.

CTAB and citric acid were used to cap the copper nanoparticles which could also slow down the particle growth [27, 28]. More particle growth and stability was observed in CTAB capped samples. From the TEM analysis (Fig. 5) it was clear that copper-copper oxide quantum dots of size approximately 3-5 nm were formed inside the colloidal solution with a shell thickness approximately $1 \mathrm{~nm}$. Citric acid forms larger clusters with cop- per nanoparticles helped in forming copper citrate, which increased the viscosity of the solution. This may be due to the excess citric acid which formed complex citrate structures, that were removed by centrifugation.

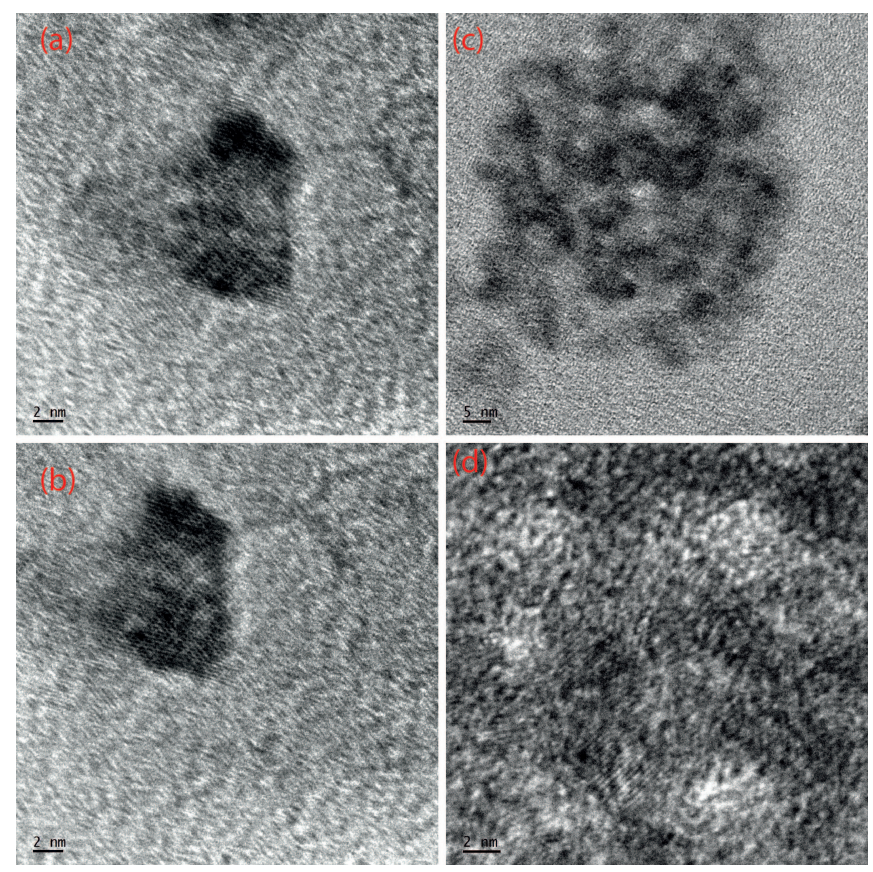

FIG. 5. (a) and (b) HRTEM images of CTAB coated coppercopper oxide core-shell quantum dots. It shows very good surface morphology and lattice planes clearly, (c) and (d) HRTEM images of citric acid coated copper-copper oxide core-shell quantum dots. The size of the quantum dots varied from $2-5 \mathrm{~nm}$. It also shows very good surface morphology. It also shows its lattice planes clearly.

Optical characterization and analysis: Fig. 6 shows the UV-Visible spectra of copper-copper oxide core-shell nanoparticles. From this graph, it is found that when the concentration of the reducing agent $\left(\mathrm{NaBH}_{4}\right)$ was increased, the SPR peak was shifted to lower wavelength region, due to the formation of larger nanoparticles. At lower concentration of the reducing agent, the SPR peak was obtained at higher wavelength region due to the formation of copper-copper oxide core-shell quantum dots. Optical absorption spectra directly depend on the particle size of the nanoparticles. This is regarded as an extrinsic size effect. In visible wavelength, the $\mathrm{SPR}$ is different for different metallic nanoparticles due to differing dielectric properties originated by the small overlap between the SPR and the interband transition. Copper has a completely filled $3 \mathrm{~d}$ orbital and just one electron in the $4 \mathrm{~s}$ orbital. The interband transition occurs within the broad conduction band, and hence the SPR peak is highly broad and the interband transitions practically overlap with SPR. This results in the decrease in SPR intensity and increase in SPR width. The resonance properties depend on the properties of dispersing medium and particles because they polarize each other and the charge density has to adjust not only to the in- 
cident fields but also the field caused by polarization[16]. The change in the dielectric medium in which nanoparticles are dispersed also affects the spectral position of the plasmon resonance. An increase in the dielectric function of the surrounding medium causes the plasmon resonance position to shift to longer wavelength. The experiments were carried out in room temperature $\left(27^{\circ} C\right)$. Here water is the dispersion medium and when the temperature increases, the refractive index of water decreases. If we change the temperature of the surrounding medium, the SPR will also shift because the refractive index is a function of temperature. Thus we can say that the temperature is an important factor for SPR peak shifting.
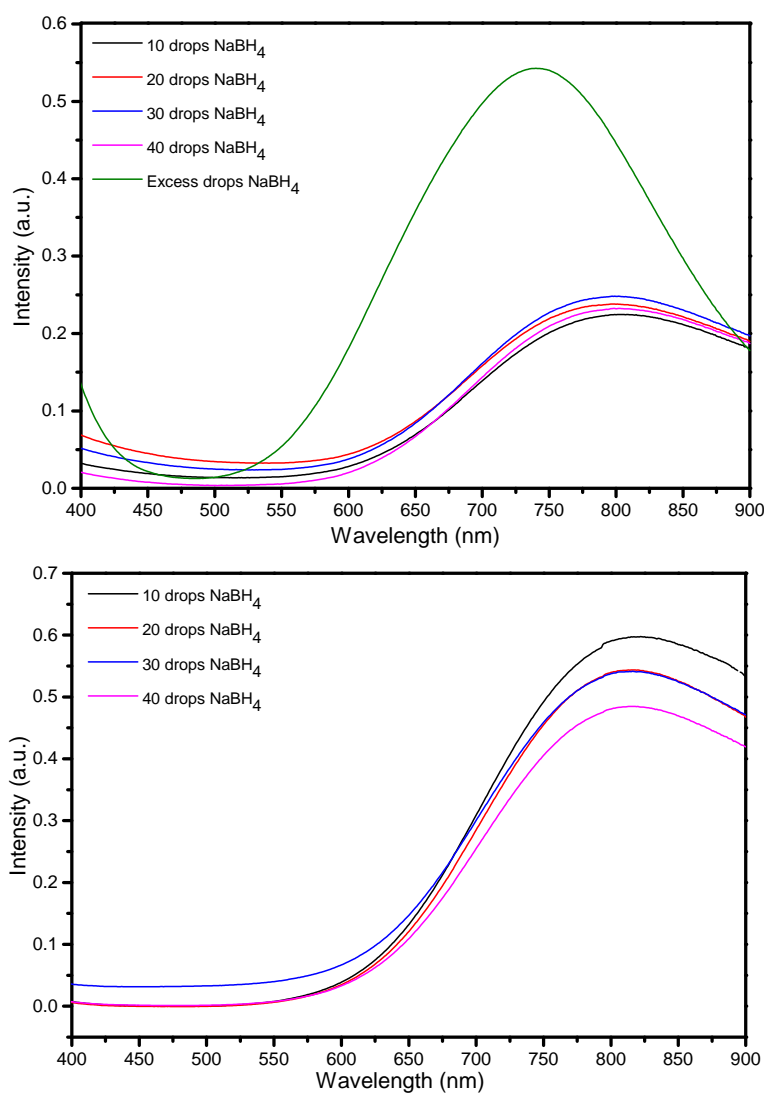

FIG. 6. UV-Visible spectra of copper-copper oxide core-shell nanoparticles are shown. a) represents the absorbance spectra of citric acid coated quantum dots, b) represents the CTAB coated quantum dots. In both cases the SPR is obtained at the near infra-red range approximately 780 - $840 \mathrm{~nm}$.

The surface curvature is another important factor affecting the rigidity of the plasmon resonance, causing the observed energy shift. With a very low surface curvature (i.e. particle size is larger), the surface plasmon becomes stiffer because the orbitals are overlapped to a greater degree resulting in a higher energy resonance and hence a blue-shift was observed for larger particles. With a high surface curvature (i.e. small particle size), the surface plasmon becomes looser because the orbitals have more space for movement (they are slightly farther apart) resulting in a lower energy resonance and therefore the
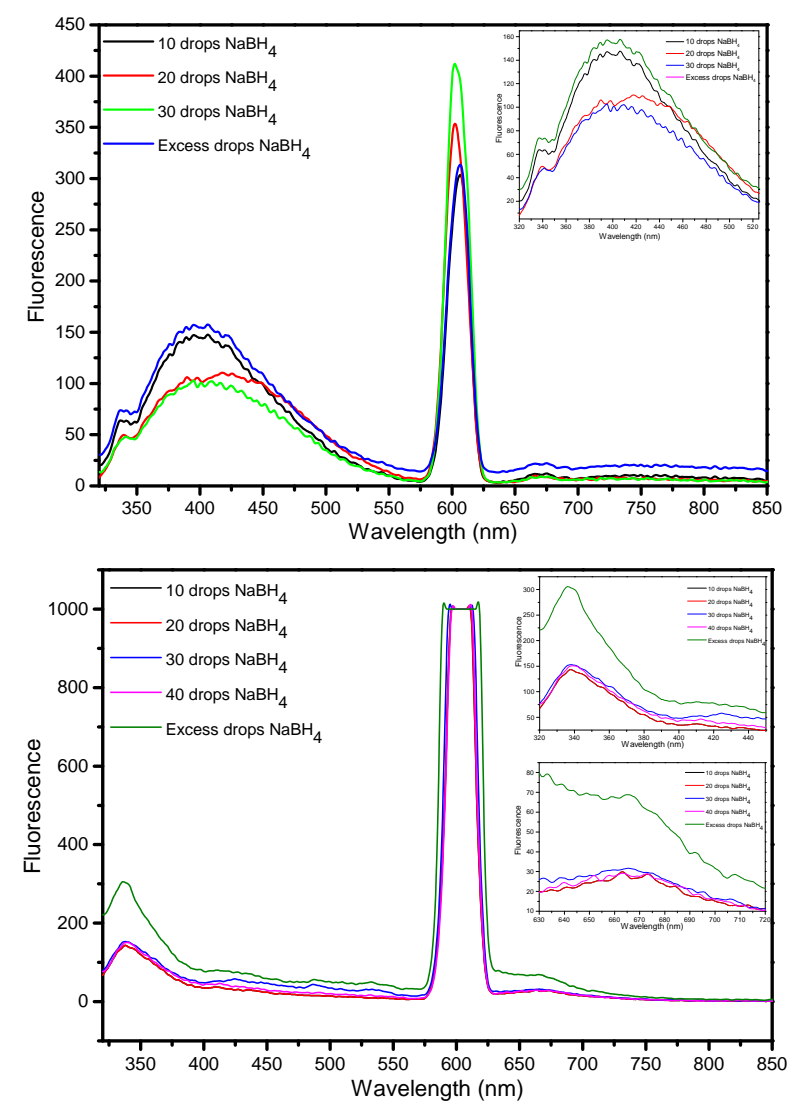

FIG. 7. Photoluminescence spectra of copper-copper oxide core-shell quantum dots. a) represent citric acid coated quantum dot, b) represents CTAB coated quantum dot. In both cases it shows one strong $U V$ emission and one weak visible emission for the excitation wavelength $300 \mathrm{~nm}$.

red-shift was observed for smaller particles. Also, the electron wave functions were modified, whereby the electron distribution and polarizability near the surface may also be changed[29].

Fig. 7 shows the photoluminescence spectra of coppercopper oxide core-shell quantum dots. For the excitation wavelength of $300 \mathrm{~nm}$, for all samples, two peaks were observed, one broader and the second narrower and sharper. When the particle size decreased, the surface curvature increased, the first emission peak (the UV emission) was shifted towards the higher wavelength region and for the second peak (visible emission-the sharp peak) shifted towards the lower wavelength region. For an excitation wavelength of $300 \mathrm{~nm}$, at lower excitation wavelengths we got broad peaks and at higher wavelengths, we got sharp peaks for the same particle size. The intensity of the peak depends on the lifetime of the process, i.e. the average time the electron stays in its excited state before emitting a photon. For less intense peaks lifetime was less and the reverse. Another reason for the less intense peaks was the recombination of bound excitons. The UV emission was due to the recombination of bound excitons. The bound exciton is usually an electron-hole pair bound 
to a defect such as a lattice atom vacancy. It gives additional coulomb energy to make the bound exciton energy lower than that of the free neutral exciton giving redshift in PL peak and broadening of peak width. This is the reason for UV emission peak broadening and redshift $[30,31]$.

Fig. 8a shows the computed extinction spectra for the copper nanosphere of radius $3 \mathrm{~nm}$. A resonance peak was observed in the $\mathrm{IR}$ region (at $870 \mathrm{~nm}$ ). Fig. $8 \mathrm{~b}$ shows the corresponding electric field intensity in the vicinity of the nanoparticle for the wavelength of $870 \mathrm{~nm}$. From the figure, it was observed that the resonance peak is due to the dipole localized surface plasmon mode induced on the nanoparticle.
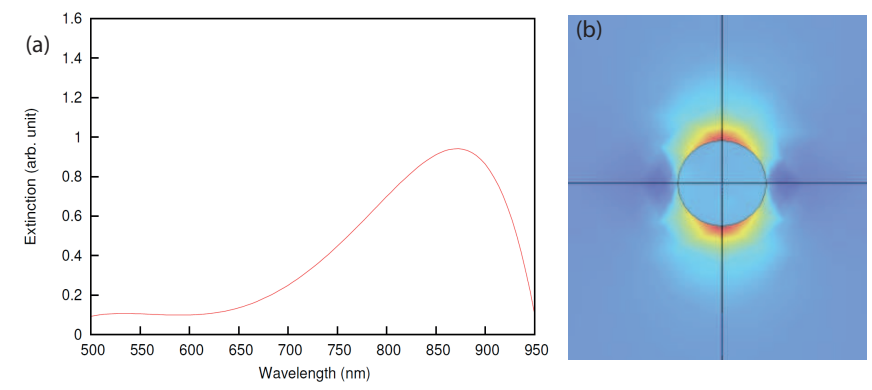

FIG. 8. Theoretical simulation results. a) Extinction spectra for a copper nano sphere of size $3 \mathrm{~nm}$ immersed in water. b) The electric field intensity at wavelength $870 \mathrm{~nm}$ corresponding to the peak in extinction spectra.

From the theoretical simulation results, SPR of copper quantum dots were at $870 \mathrm{~nm}$ while the experimental value of the SPR obtained at $820 \mathrm{~nm}$. The shift in SPR of experimental value from the theoretical value was due to the formation of copper oxide shell in the surface, the non-uniform shape of the surface and the random orientation of copper quantum dots in the ensemble that we consider. By increasing the size of the nanoparticles, we can tune the SPR into the visible range from the near infrared region which assumes great significance due to their promising application potential in SPR based biosensors which exploits the visible range SPR in copper nano col- loids, and thus can emerge as a viable substitute for the gold and silver-based biosensors[32].

We can functionalize the surface of the copper-copper oxide core-shell nanoparticles using suitable chemical compounds. For that, the solution can be treated with a peptide of the general formula, for example, Cys $(A A)_{n}-$ Cys (where AA is any amino acid and Cys is cysteine), the colour of the solution will change due to the aggregation of nanoparticles. This aggregation of nanoparticles cause a change in SPR shift. This is due to the presence of the peptide bond. If we hydrolyze this peptide bond using suitable chemical compounds, it will separate and we will get a shift is SPR peak opposite to the previous case. Using this methodology we can demonstrate the copper-based biosensors [32, 33].

\section{CONCLUSION}

Copper-copper oxide core-shell quantum dots were successfully synthesized using the chemical reduction method. The size reduction led to remarkable changes in their optical responses as compared to the bulk metal. This response depends on the size, shape and dielectric environment of the nanoparticles. Quantum confinement led to a modification in the dielectric constant of the confined metal at small particle sizes. It leads to the broadening of SPR upon its size reduction. The tuning of SPR resonance towards the near IR-visible region can make the copper-copper oxide core-shell nanoparticles as an ideal substitute for gold and silver nanoparticles that are widely used for biosensor applications.

\section{ACKNOWLEDGEMENT}

Swapna Nair acknowledges University Grants Commission (UGC, India) for the funding (UGC BSR Start-up F.20-1/2013) and Department of Science and Technology (DST, Govt. of India, Fast Track - YSS/2014/000436) and Department of Biotechnology, Govt. of India. Prabhash acknowledges Kerala State Council for Science Technology and Environment (Ref: No. 001/FSHPMAIN/2015/KSCSTE) for the research fellowship.
[1] K. L. Kelly, E. Coronado, L. L. Zhao, and G. C. Schatz, The Journal of Physical Chemistry B 107, 668 (2003).

[2] I. O. Sosa, C. Noguez, and R. G. Barrera, The Journal of Physical Chemistry B 107, 6269 (2003).

[3] B. R. Cuenya, Thin Solid Films 518, 3127 (2010).

[4] A. Lazarides, K. L. Kelly, T. Jensen, and G. Schatz, Journal of Molecular Structure: THEOCHEM 529, 59 (2000).

[5] P. K. Jain, X. Huang, I. H. El-Sayed, and M. A. ElSayed, Plasmonics 2, 107 (2007).

[6] A. Moores and F. Goettmann, New Journal of Chemistry 30, 1121 (2006).

[7] T. K. Sau, A. L. Rogach, F. Jäckel, T. A. Klar, and J. Feldmann, Advanced Materials 22, 1805 (2010).
[8] R. R. Arvizo, S. Bhattacharyya, R. A. Kudgus, K. Giri, R. Bhattacharya, and P. Mukherjee, Chemical Society Reviews 41, 2943 (2012).

[9] S. Eustis and M. A. El-Sayed, Chemical Society Reviews 35, 209 (2006).

[10] P. K. Jain, X. Huang, I. H. El-Sayed, and M. A. ElSayed, Accounts of chemical research 41, 1578 (2008).

[11] S. Lal, S. Link, and N. J. Halas, Nature photonics 1, 641 (2007).

[12] P. Khanna, S. Gaikwad, P. Adhyapak, N. Singh, and R. Marimuthu, Materials Letters 61, 4711 (2007).

[13] G. De, L. Tapfer, M. Catalano, G. Battaglin, F. Caccavale, F. Gonella, P. Mazzoldi, and R. Haglund Jr, Applied physics letters 68, 3820 (1996). 
[14] M. Mitsushio, K. Miyashita, and M. Higo, Sensors and Actuators A: Physical 125, 296 (2006).

[15] S. Padovani, C. Sada, P. Mazzoldi, B. Brunetti, I. Borgia, A. Sgamellotti, A. Giulivi, F. dAcapito, and G. Battaglin, Journal of Applied Physics 93, 10058 (2003).

[16] S. K. Ghosh and T. Pal, Chemical Reviews 107, 4797 (2007).

[17] T. Ghodselahi, M. Vesaghi, and A. Shafiekhani, Journal of Physics D: Applied Physics 42, 015308 (2008).

[18] J.-Y. Bigot, J.-Y. Merle, O. Cregut, and A. Daunois, Physical review letters 75, 4702 (1995).

[19] M. Cortez-Valadez, J.-G. Bocarando-Chacon, A. Hernández-Martínez, R. B. Hurtado, R. A. Alvarez, J. Roman-Zamorano, J. Flores-Valenzuela, R. Gámez-Corrales, H. Arizpe-Chávez, and M. FloresAcosta, Nanoscience and Nanotechnology Letters 6, 580 (2014).

[20] L. Qi, J. Ma, and J. Shen, Journal of colloid and interface science 186, 498 (1997).

[21] Y. Kobayashi, S. Ishida, K. Ihara, Y. Yasuda, T. Morita, and S. Yamada, Colloid and Polymer Science 287, 877 (2009).

[22] B. K. Park, S. Jeong, D. Kim, J. Moon, S. Lim, and J. S. Kim, Journal of colloid and interface science 311, 417 (2007).
[23] C. Multiphysics, Inc., http://www. comsol. com (2011).

[24] D. H. Park, J. W. Kang, J. D. Luo, T. D. Kim, A. K.-Y. Jen, C. H. Lee, and W. N. Herman, in Optics $\mathcal{G}$ Photonics 2005 (International Society for Optics and Photonics, 2005) pp. 59350O-59350O.

[25] J.-P. Berenger, Journal of computational physics 114, 185 (1994).

[26] P. Kundu, C. Nethravathi, P. A. Deshpande, M. Rajamathi, G. Madras, and N. Ravishankar, Chemistry of Materials 23, 2772 (2011).

[27] L. Li, K. Mak, C. Leung, K. Chan, W. Chan, W. Zhong, and P. Pong, Microelectronic Engineering 110, 329 (2013).

[28] S. Wang, W. Lu, O. Tovmachenko, U. S. Rai, H. Yu, and P. C. Ray, Chemical physics letters 463, 145 (2008).

[29] J. Zhu, Journal of Nanoparticle Research 11, 785 (2009).

[30] I. Kriegel, C. Jiang, J. Rodrguez-Fernndez, R. D. Schaller, D. V. Talapin, E. Da Como, and J. Feldmann, Journal of the American Chemical Society 134, 1583 (2012).

[31] D. Haranath, S. Sahai, and P. Joshi, Applied Physics Letters 92, 233113 (2008).

[32] C. Guarise, L. Pasquato, V. De Filippis, and P. Scrimin, Proceedings of the National Academy of Sciences of the United States of America 103, 3978 (2006).

[33] P. Prabhash, V. Haritha, S. S. Nair, and R. Pilankatta, Sensors and Actuators B: Chemical 240, 580 (2017). 